\title{
Some applications of polarized inelastic neutron scattering in magnetism
}

\author{
B ROESSLI ${ }^{1}$ and $\mathrm{P}$ BÖNI ${ }^{2}$ \\ ${ }^{1}$ Laboratory for Neutron Scattering, ETH Zürich \& Paul Scherrer Institute, CH-5232 \\ Villigen PSI, Switzerland \\ ${ }^{2}$ Physik-Department E21, Technische Universität München, D-85747 Garching, Germany \\ E-mail: bertrand.roessli@psi.ch
}

\begin{abstract}
A brief account of applications of polarized inelastic neutron scattering in condensed matter research is given. We show that full polarization analysis is the only tool allowing to discriminate unambiguously between different magnetic modes in various magnetic materials. We show by means of recent results in the Heisenberg ferromagnet EuS that the effects of dipolar interactions can be studied on a microscopic scale. Moreover, we have found for the first time indications for the divergence of the longitudinal fluctuations below $T_{\mathrm{c}}$. In the itinerant antiferromagnet chromium we demonstrate that the dynamics of the longitudinal and transverse excitations are very different, resolving a long standing puzzle concerning the slope of their dispersion. Finally, we show that a measurement of the polarization-dependent part of the cross section of non-centrosymmetric $\mathrm{MnSi}$ proves directly that the chirality of the magnetic fluctuations is left-handed.
\end{abstract}

Keywords. Polarized inelastic neutron scattering; spin waves; spin fluctuations; spin chirality.

PACS Nos 75.25.+z; 71.20.Lp; 71.70.Ej

\section{Introduction}

Polarized neutron scattering at high-flux neutron sources has proved to be a very powerful tool for the investigation of materials in the field of condensed matter physics. Nowadays polarized neutrons find application in various research fields like studies of magnetic structures using zero-field polarimetry, determination of spin densities in applied magnetic fields, identification of the polarization of magnetic fluctuations and their different modes, investigation of magnetic multi-layered structures, depolarization of neutron beams in magnetic materials, separation of coherent and incoherent scattering in hydrogen containing materials, high-resolution diffraction and spectroscopy using spin-echo techniques, not to forget applications in fundamental physics to test time-invariance properties of neutrons etc. In this short note, we provide a brief overview of recent experiments using the longitudinal polarization technique (also called full-polarization analysis), that were recently performed at the Institut Laue-Langevin and at the Swiss Spallation Source SINQ 
using cold and thermal neutron-triple axis spectrometers. Of course, due to limitations in space we cannot give a complete review of the neutron polarization techniques. Therefore, we refer the interested reader to recent reviews and conference proceedings on the subject [1].

\section{Longitudinal-polarization analysis technique}

The method of the longitudinal-polarization analysis for both elastic and inelastic neutron scattering was introduced by Moon, Riste and Koehler at Oak Ridge National Laboratory [2]. The original principle works as follows: The incident neutron beam is polarized by means of a polarizing monochromator. After scattering, the neutrons are reflected from a polarizing analyzer before being detected in a neutron counter. Of course the neutrons can also be polarized by other means like reflection from polarizing supermirrors or by transmission through polarized ${ }^{3} \mathrm{He}$. By use of spin-flippers, the polarization of the incident and scattered neutrons can be selected to be either parallel or anti-parallel to the guide field $\left(B_{\mathrm{g}} \simeq 1 \mathrm{mT}\right)$ that defines the quantization axis of the neutron spin along the flight path from the polarizer to the analyzer.

This set-up allows to determine four different cross-sections $\sigma^{++}, \sigma^{--}, \sigma^{+-}$and $\sigma^{-+}$. The indices,+- refer to the polarisation $\mathbf{P}$ of the neutrons being parallel or anti-parallel to the magnetic field $\mathbf{B}$, respectively, so that ++ and -- scattering is non spin-flip, whereas +- and -+ events are spin-flip. At the sample position, a magnetic field can be applied either along or perpendicular to the scattering vector Q. In contrast to the recently developed technique of three-dimensional polarimetry that is performed in zero field at the sample position [3], the Moon-Riste-Koehler set-up that we discuss in this paper allows only to measure the polarization along the applied magnetic field.

The neutron cross-section for neutron scattering contains three contributions: (1) a pure nuclear contribution that gives rise to Bragg, phonon and isotopic incoherent scattering, (2) a pure magnetic contribution due to magnetic Bragg scattering and inelastic magnetic scattering, as well as spin-incoherent scattering, (3) a magneticnuclear interference term. An elastic contribution to the interference term appears if nuclear and magnetic Bragg peaks super-impose. This happens in ferromagnetic and some antiferromagnetic materials. An inelastic contribution of the same kind may appear only in special cases, for example when the spin-lattice interaction in a magnetic material cannot be neglected (e.g. invar alloys, Spin-Peierls systems). We point out that this term cannot be measured by means of longitudinal-polarization analysis.

Since we present below exclusively examples of inelastic neutron scattering from magnetic materials we quote only the matrix elements for magnetic scattering:

$$
\begin{aligned}
& \left\langle+\left|\boldsymbol{\sigma} \cdot \mathbf{D}_{\perp}\right|+\right\rangle=D_{\perp z}, \\
& \left\langle-\left|\boldsymbol{\sigma} \cdot \mathbf{D}_{\perp}\right|-\right\rangle=-D_{\perp z}, \\
& \left\langle-\left|\boldsymbol{\sigma} \cdot \mathbf{D}_{\perp}\right|+\right\rangle=D_{\perp x}+i D_{\perp y}, \\
& \left\langle+\left|\boldsymbol{\sigma} \cdot \mathbf{D}_{\perp}\right|-\right\rangle=D_{\perp x}-i D_{\perp y} .
\end{aligned}
$$

$\boldsymbol{\sigma}$ is the operator of the neutron magnetic moment and $\mathbf{D}_{\perp}=\hat{\mathbf{Q}} \times(\rho(\mathbf{Q}, \omega) \times \hat{\mathbf{Q}})$ 


\section{Polarized INS in magnetism}

is the magnetic interaction vector implying that only magnetic fluctuations perpendicular to $\mathbf{Q}$ contribute to the scattering cross-section. $\rho(\mathbf{Q}, \omega)$ is the Fourier transform in space and time of the magnetic moment distribution $\mathbf{M}(\mathbf{r}, t)$. From the above equations two important applications emerge. First, if the neutron polarization of the incident beam $\mathbf{P}$ (i.e. $\boldsymbol{\sigma}$ ) is aligned along the scattering vector $\mathbf{Q}$, then all the magnetic scattering is spin-flip. This allows an unambiguous separation of the often weak magnetic scattering, e.g. from phonons. Second, in a magnetic field perpendicular to $\mathbf{Q}$, i.e. $\mathbf{P}$, spin fluctuations (spin waves or paramagnetic fluctuations) with components along $\mathbf{P}$ occur in the NSF channel, while spin fluctuations with components perpendicular to $\mathbf{P}$ occur in the SF channel.

\subsection{Transverse and longitudinal excitations in the dipolar ferromagnet EuS}

The magnetic properties of compounds with localized spin densities can be conveniently described on the basis of the Heisenberg Hamiltonian $H=-\sum_{i, j} J_{i j} \mathbf{S}_{\mathbf{i}} \cdot \mathbf{S}_{\mathbf{j}}$, where $J_{i j}$ is the exchange integral between the spins located at the $i$ - and $j$-position, respectively. Depending on the sign of the exchange integral $\mathbf{J}(\mathbf{q})$, either an antiferromagnetic or a ferromagnetic ground-state is favored. If exchange interactions extend beyond nearest neighbors, competing effects can occur that may lead to noncollinear or even incommensurate magnetic structures. The dipolar interactions can be included in the Hamiltonian for an isotropic ferromagnet by adding the dipolar operator $H_{\mathrm{d}}=\sum_{\alpha, \beta, i, j} G\left(\frac{\delta^{\alpha \beta}}{r_{i, j}^{3}}-\frac{3 r_{i, j}^{\alpha} r_{i, j}^{\beta}}{r_{i, j}^{5}}\right) S_{i}^{\alpha} S_{j}^{\beta}$. Because the dipolar interactions are long-range, they affect the spin-wave dynamics only at small momentum transfers $q<q_{\mathrm{d}}$, where the dipolar wavenumber is defined by $D q_{\mathrm{d}}^{2}=g \mu_{0} \mu_{\mathrm{B}} M$. Here, $D$ is the stiffness of the spin waves. For the model Heisenberg antiferromagnet EuS one obtains $q_{\mathrm{d}}=0.245 \AA^{-1}$. Obviously, $q_{\mathrm{d}}$ is a measure of the relative strength of the dipolar to the exchange interactions. The dipolar terms destroy the isotropy of the Hamiltonian and in the ordered phase, one has to distinguish between magnetic fluctuations parallel and perpendicular (= spin waves) to the magnetization $\mathbf{M}$, leading to several magnetic modes below $T_{\mathrm{c}}$. Hence, spin excitations $\delta \mathbf{S}^{\mathrm{L}}$ along (longitudinal, L) and $\delta \mathbf{S}^{\mathrm{T}}$ transverse (T) to the reduced momentum transfer $\mathbf{q}$ are no longer identical. If the magnetic field is applied along the vertical direction, two spin wave modes (i.e. L and T) as well as a longitudinal mode with a polarization along $\mathbf{M}$ can be identified by means of polarization analysis. As an example, we show in figure 1 typical constant- $\mathbf{Q}$ scans that were measured at the positions $(1.82,0,0)$ and $(2,0.18,0)$ in reciprocal space. It is clearly seen that the spin-waves occurring in the SF channel have a very different intensity. The reason is that the spin waves with L-polarization attain a mass due to demagnetization that occurs only for spin deviations $\delta \mathbf{S} \| \mathbf{q}$. In other words, it is only the spin waves with $T$ polarization that show Goldstone behavior $1 / q^{2}$. These results can be summarized by the following expressions for the wavelength-dependent susceptibility $(\alpha=L, T)$ $\chi_{\alpha}(\mathbf{Q}) \propto \frac{q_{d}^{2}}{q^{2}+\delta_{\alpha, \mathrm{L}} q_{\mathrm{d}}^{2}}$, showing that the number of Goldstone modes is reduced from two to one. Of course, close to $T_{\mathrm{c}}$ the dynamics becomes also different for the two modes.

The NSF channels contain the spin fluctuations parallel to the magnetization, i.e. $\chi_{z}(\mathbf{Q}, \omega)$. They become very strong close to $T_{\mathrm{c}}$ since they are due to two 


\section{B Roessli and P Böni}

magnon processes. Within our scattering geometry, the results at the two Qpositions are identical as expected. We have investigated the $T$-dependence of the energy integrated susceptibility $\chi_{z}(\mathbf{Q})$ in detail and find clear deviations from the Lorentzian behavior $1 /\left(q^{2}+\kappa_{z}^{2}\right)$, as shown in figure 1 . Here, $\kappa_{z}$ designates the inverse correlation length. This is the first time that indications for the divergence of the longitudinal susceptibility $\chi_{z}(\mathbf{Q}) \propto 1 / q$ for $q \rightarrow 0$ have been found [4].
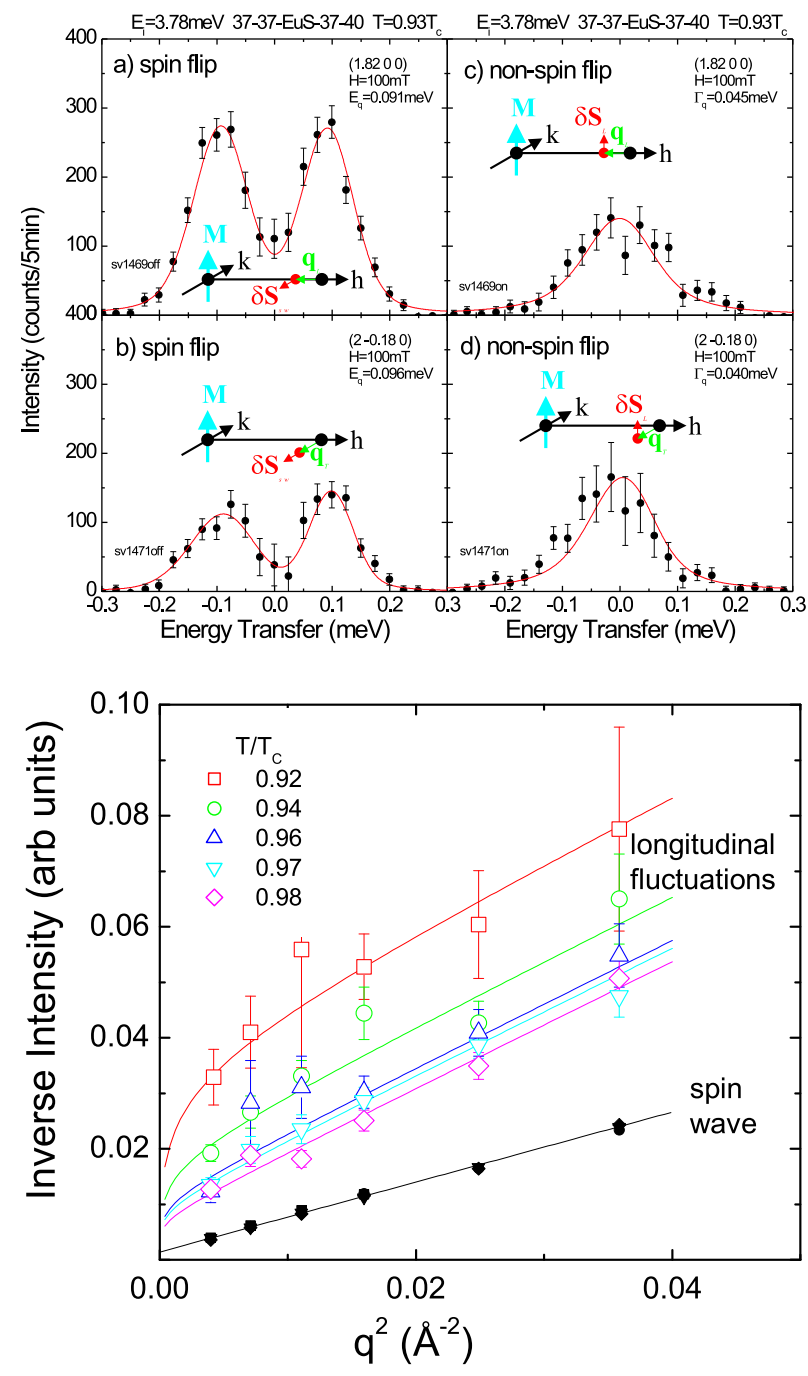

Figure 1. Top: Constant-Q scans probing the magnetic fluctuations at $(1.82,0,0)$ and $(2,0.18,0)$. The quadrants show the polarization of the magnetic modes with respect to $\mathbf{M}$ and $\mathbf{q}$. Bottom: Inverse of the integrated intensities of the spin wave and longitudinal spin fluctuations versus $q^{2}$. The latter clearly deviate at small $q$ from Lorentzian behavior indicating the $1 / q$-divergence due to the Goldstone mode. 


\subsection{Polarization dependence of the spin-density-wave excitations of chromium}

Chromium is one of the most fascinating itinerant antiferromagnets exhibiting a linearly polarized incommensurate magnetic ground state [5]. Cr undergoes a transition from the paramagnetic phase at $T_{\mathrm{N}}=311 \mathrm{~K}$ to a spin-density wave phase characterized by the propagation vector $\mathbf{Q}_{\mathbf{0}}=(1 \pm \delta, 0,0)$ with $\delta=0.048$. The incommensurate magnetic order is the result of the nesting properties of the electron and hole Fermi surface. In the transverse spin-density wave (TSDW) phase $T_{s f}=121 K<T<T_{\mathrm{N}}$ the magnetic moments are aligned perpendicular to $\mathbf{Q}_{\mathbf{0}}$. At $T_{s f}$ the magnetic structure undergoes a first-order phase transition to a longitudinal spin-density wave (LSDW) phase with the spins aligned along $\mathbf{Q}_{\mathbf{0}}$.

Although experimentally well-studied, the nature of the magnetic excitations of $\mathrm{Cr}$ are poorly understood. First of all, the magnetic excitations that emerge from the satellite positions have an extremely high velocity $c=1020 \mathrm{meV} \AA^{-1}$. Second, it has been found that in the TSDW phase the longitudinal magnetic fluctuations are strongly enhanced for energy transfers below $\sim 8 \mathrm{meV}$. While the spin-wave modes are not affected by the transition at $T_{s f}$, additional excitations were reported in the TSDW phase between the incommensurate positions $(1 \pm \delta, 0,0)$ with a well-defined maximum near $4 \mathrm{meV}$ at the incommensurate position $\mathbf{Q}=(\mathbf{1}, \mathbf{0}, \mathbf{0})$ [6]. Figure 2 shows a contour plot of the inelastic intensity recorded on the tripleaxis TASP in the TSDW-phase at $230 \mathrm{~K}$ [7]. Most dominant are the very steep excitations that emerge from the incommensurate positions. Another important feature is the Fincher mode near $4 \mathrm{meV}$ mentioned above and the additional scattering near $(1.02,0,0)$ and $3 \mathrm{meV}$ that has no counterpart at the symmetric position $(0.98,0,0)$. This unexpected asymmetry was recently confirmed on another $\mathrm{Cr}$ crystal [8].

In order to obtain information about the polarization of the magnetic modes we have measured the SF and NSF-cross-sections in Cr. The crystal was cooled through $T_{\mathrm{N}}$ in a magnetic field of $20 \mathrm{~T}$ to induce a single- $\mathbf{Q}$ state. During the experiments, a vertical field $\mathbf{B}_{z}=4 \mathrm{~T}$ along $[0,0,1]$ was applied to enforce a singledomain state with the magnetic moments lying in the $(h, k, 0)$ scattering plane. Hence, the SF- and NSF-scattering is due to longitudinal and transverse fluctuations, respectively. Figure 2 shows constant- $E$ scans that have been performed with polarization analysis [9]. The peaks at the incommensurate positions correspond to cuts through the rods of inelastic scattering that emerge from the magnetic Bragg peaks. The longitudinal polarization of the Fincher mode is clearly established. The solid lines are fits to Gaussian functions. It is seen that there is significant polarization-independent 'commensurate' scattering around $(1,0,0)$. The fits clearly show that the width of the incommensurate scattering with longitudinal polarization is broader than the scattering with transverse polarization. The broadening of the L-peaks with respect to the T-peaks is compatible with the predictions of Fishman and Liu [10]. Namely, the velocity $c_{\mathrm{L}}$ of the longitudinal modes is clearly smaller than the velocity $c_{\mathrm{T}}$ of the transverse modes. In particular, the width of the transverse peaks is now in good agreement with the prediction of the three band model $c_{\mathrm{T}}=v_{\text {Fermi }} / \sqrt{3}=1500 \mathrm{meV}[10]$. 

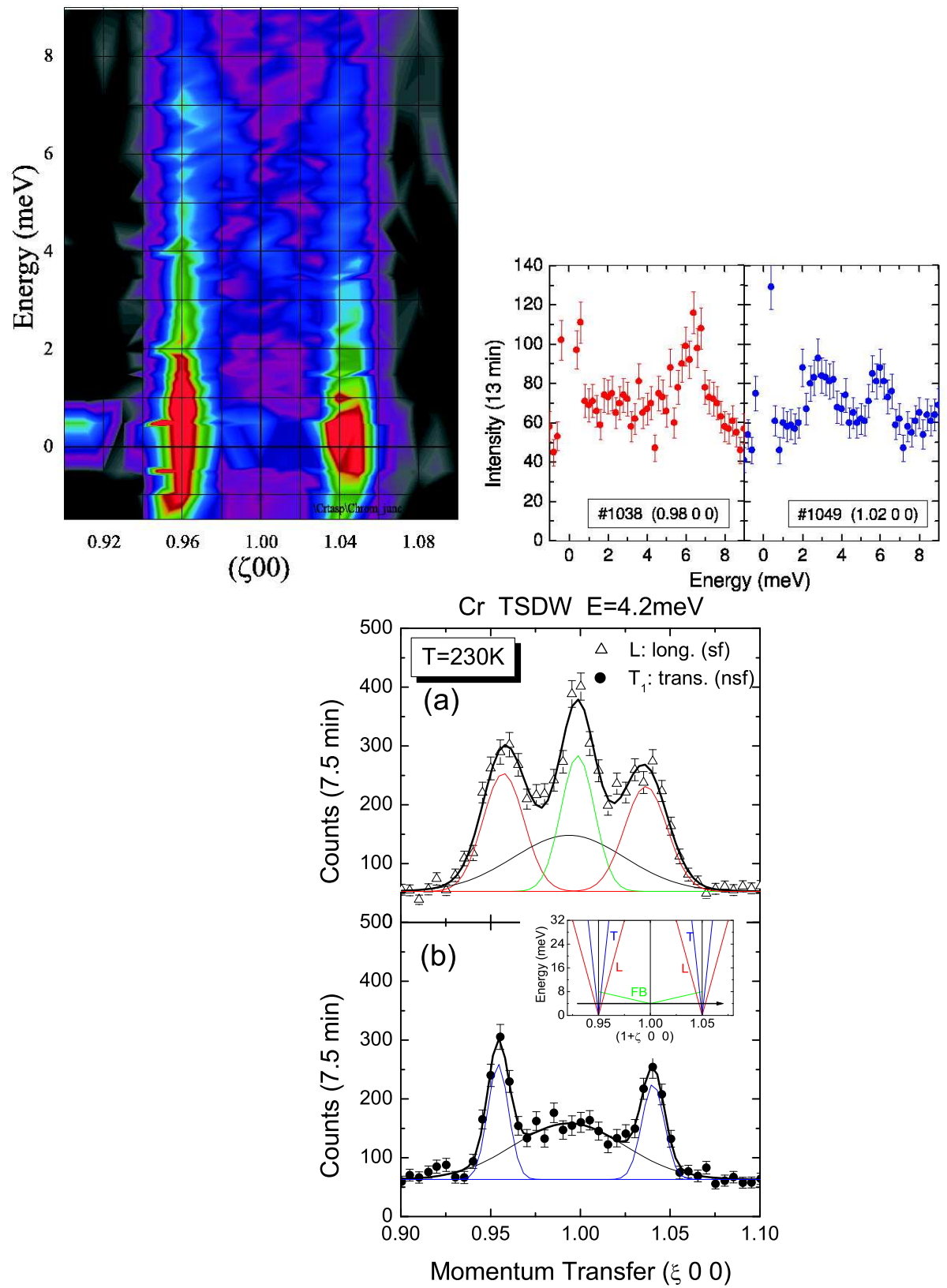

Figure 2. Left: Contour map of the excitation spectrum as measured in the transverse spin density wave phase of $\mathrm{Cr}$ at $T=230 \mathrm{~K}$. The intense peak near $4 \mathrm{meV}$ and $\mathbf{Q}=(1,0,0)$ is the Fincher mode. Right: Note the additional peak near $(1.02,0,0)$ and $3 \mathrm{meV}$ that has no counterpart at $(0.98,0,0)$. Bottom: Constant- $E$ scans at $4.2 \mathrm{meV}$ probing the longitudinal and transverse fluctuations along the $[1,0,0]$ direction in the TSDW phase at $T=230 \mathrm{~K}$ with polarization analysis. 


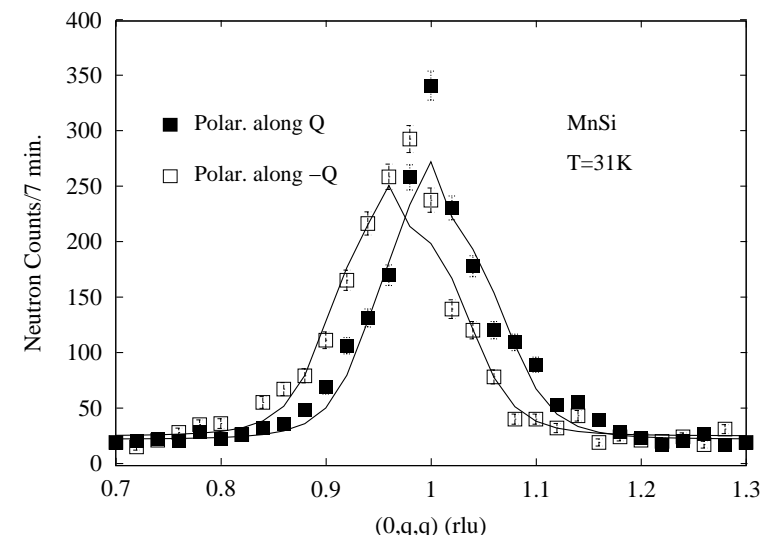

Figure 3. Inelastic spectra in $\mathrm{MnSi}(\hbar \omega=0.5 \mathrm{meV})$ at $T=31 \mathrm{~K}\left(T_{\mathrm{N}}=29.5\right.$ $\mathrm{K}$ ) for the neutron polarization parallel and anti-parallel to the scattering vector, respectively. See text for details.

\subsection{Chiral fluctuations in non-centrosymmetric MnSi}

In the previous examples we have demonstrated that longitudinal polarization analysis allows to determine unambiguously the polarization of the magnetic modes in magnetic materials. In the following, we show that chiral fluctuations can be measured in zero-field by means of inelastic polarized neutron scattering in compounds with non-centrosymmetric symmetry. Namely, the crosssection of magnetic scattering with polarized neutrons is proportional to $\frac{\mathrm{d}^{2} \sigma}{\mathrm{d} \Omega \mathrm{d} \omega} \sim$ $\sum_{\alpha, \beta}\left(\delta_{\alpha, \beta}-\hat{Q}_{\alpha} \hat{Q}_{\beta}\right) A^{\alpha \beta}(\mathbf{Q}, \omega)+\sum_{\alpha, \beta}\left(\hat{\mathbf{Q}} \cdot \mathbf{P}_{0}\right) \sum_{\gamma} \epsilon_{\alpha, \beta, \gamma} \hat{Q}^{\gamma} B^{\alpha \beta}(\mathbf{Q}, \omega)$, where $(\mathbf{Q}, \omega)$ designates the momentum and energy transfer from the neutron to the sample and $\hat{\mathbf{Q}}=\mathbf{Q} /|\mathbf{Q}|$. The indices $\alpha, \beta, \gamma$ indicate Cartesian coordinates. The first term in the cross-section is independent of the polarization of the incident neutrons, while the second is polarization dependent through the factor $\left(\hat{\mathbf{Q}} \cdot \mathbf{P}_{0}\right)$. $\mathbf{P}_{0}$ denotes the direction of the neutron polarization and its scalar is equal to 1 when the beam is fully polarized. $A^{\alpha \beta}$ and $B^{\alpha \beta}$ are the symmetric and antisymmetric parts of the scattering function $S^{\alpha \beta}, A^{\alpha \beta}=\frac{1}{2}\left(S^{\alpha \beta}+S^{\alpha \beta}\right)$ and $B^{\alpha \beta}=\frac{1}{2}\left(S^{\alpha \beta}-S^{\alpha \beta}\right) . \quad S^{\alpha \beta}$ are the Fourier transforms of the spin correlation function $\left\langle s_{l}^{\alpha} s_{l^{\prime}}^{\beta}\right\rangle$, i.e. $S^{\alpha \beta}(\mathbf{Q}, \omega)=\frac{1}{2 \pi N} \int_{-\infty}^{\infty} \mathrm{d} t \mathrm{e}^{-i \omega t} \sum_{l l^{\prime}} \mathrm{e}^{i \mathbf{Q} \cdot\left(\mathbf{X}_{l}-\mathbf{X}_{l^{\prime}}\right)}\left\langle s_{l}^{\alpha} s_{l^{\prime}}^{\beta}(t)\right\rangle$. The vectors $\mathbf{X}_{l}$ designate the positions of the scattering centers in the lattice. Following ref. [11] we now define an axial vector $\mathbf{B}$ by $\sum_{\alpha \beta} \epsilon_{\alpha \beta \gamma} B^{\alpha \beta}=B^{\gamma}(\mathbf{Q}, \omega)$. $\mathbf{B}$ characterizes the antisymmetric part of the susceptibility. The corresponding cross-section for magnetic scattering which depends on the neutron polarization is proportional to $\left(\hat{\mathbf{Q}} \cdot \mathbf{P}_{0}\right)(\hat{\mathbf{Q}} \cdot \mathbf{B})$. This term vanishes for a centro-symmetric system.

In order to prove this statement we show in figure 3 a constant- $E$ scan conducted in the weak-ferromagnet $\mathrm{MnSi}$ at an energy transfer $\hbar \omega=0.5 \mathrm{meV}$ measured in the paramagnetic phase using a polarized beam. It is obvious from figure 3 that the quasielastic scattering is polarization-dependent when the polarization of the neutrons is chosen along $\pm \mathbf{Q}$. Of particular importance, we find that the neutron peaks appear at positions incommensurate with respect to the chemical lattice, 


\section{B Roessli and P Böni}

namely at $\mathbf{Q}=\boldsymbol{\tau} \pm \boldsymbol{\delta}$ ( $\boldsymbol{\tau}$ is a reciprocal lattice vector). We emphasize that this shift from the commensurate position is not visible in an experiment with unpolarized neutrons or when the polarization of the neutron beam is chosen like $\mathbf{P} \perp \mathbf{Q}$ ( $\rightarrow$ $\left.\hat{\mathbf{Q}} \cdot \mathbf{P}_{0}=0\right)$ because of the intrinsic width of the scattering [12]. Because the crystal structure of $\mathrm{MnSi}$ is non-centrosymmetric and the magnetic ground-state forms a helix, we conclude that the antisymmetric part of the dynamical susceptibility is due to the Dzyaloshinskii-Moriya interaction with a uniform $\mathbf{D}$-vector. In that case, the neutron cross-section depends on the polarization of the neutron beam $[13]$ as $\left(\frac{\mathrm{d}^{2} \sigma}{\mathrm{d} \Omega \mathrm{d} \omega}\right)_{p} \sim(\mathbf{D} \cdot \hat{\mathbf{Q}})\left(\hat{\mathbf{Q}} \cdot \mathbf{P}_{0}\right) \Im \frac{\left(\chi^{\perp}(\mathbf{q}-\boldsymbol{\delta}, \omega)-\chi^{\perp}(\mathbf{q}+\boldsymbol{\delta}, \omega)\right)}{2 D}$. As shown in figure 3, using for the dynamical susceptibility $\chi^{\perp}\left(\mathbf{Q}_{\mathbf{0}}+\mathbf{q}, \omega\right)=\chi\left(\mathbf{Q}_{\mathbf{0}}+\mathbf{q}\right) /\left(\mathbf{1}-\mathbf{i} \omega / \boldsymbol{\Gamma}_{\mathbf{Q}_{\mathbf{0}}+\mathbf{q}}\right)$ reproduces the inelastic neutron data well.

\section{Conclusion}

From the examples presented here, we have shown that polarised neutrons can yield unique information on the spin dynamics of magnetic materials that cannot be obtained by any other methods. We would like to point out that using the method of longitudinal polarimetry as introduced by Moon, Riste and Koehler does not give access to the nuclear-magnetic interference term in the inelastic cross-section. It is expected that application of neutron polarimetry in zero field using inelastic neutron scattering will open new perspectives in systems where e.g. spin-lattice interactions cannot be neglected.

\section{References}

[1] S V Maleev, Phys. Usp. 45, 569 (2002)

B Roessli and P Böni, in Scattering and inverse scattering in pure and applied science edited by R Pike and P Sabatier (Academic Press, 2002) 1243; Proceedings of the PNCMI-conference, published in Physica B297 (2001)

[2] R M Moon, T Riste and W C Koehler, Phys. Rev. B181, 920 (1969)

[3] P J Brown, J B Forsyth and F Tasset, Proc. R. Soc. London A442, 147 (1993) F Tasset et al, Physica B267-268, 69 (1999)

[4] P Böni, B Roessli, D Görlitz and J Kötzler, Phys. Rev. B65, 14434 (2002)

[5] E Fawcett, Rev. Mod. Phys. 60, 209 (1988)

[6] C R Fincher, G Shirane and S A Werner, Phys. Rev. B24, 1312 (1981)

[7] P Böni, E Clementyev, C Stadler, B Roessli, G Shirane G and S A Werner, Appl. Phys. A74, S716 (2002)

[8] H Hiraka et al, Phys. Rev. B, submitted

[9] P Böni, B J Sternlieb, G Shirane, B Roessli, J E Lorenzo and S A Werner, Phys. Rev. B57, 1057 (1998)

[10] R S Fishman and S H Liu, Phys. Rev. Lett. 76, 2398 (1996); Phys. Rev. B54, 7252 (1996)

[11] S W Lovesey and G I Watson, J. Phys. Condens. Matter 10, 6761 (1998)

S W Lovesey and E Balcar, Physica B267-268, 221

[12] B Roessli, P Böni, W E Fischer and Y Endoh, Acta Phys. Pol. B34, 1557 (2003)

B Roessli, P Böni, W E Fischer and Y Endoh, Phys. Rev. Lett. 88, 237204 (2002)

[13] D N Aristov and S V Maleyev, Phys. Rev. B62, R751 (2000) 